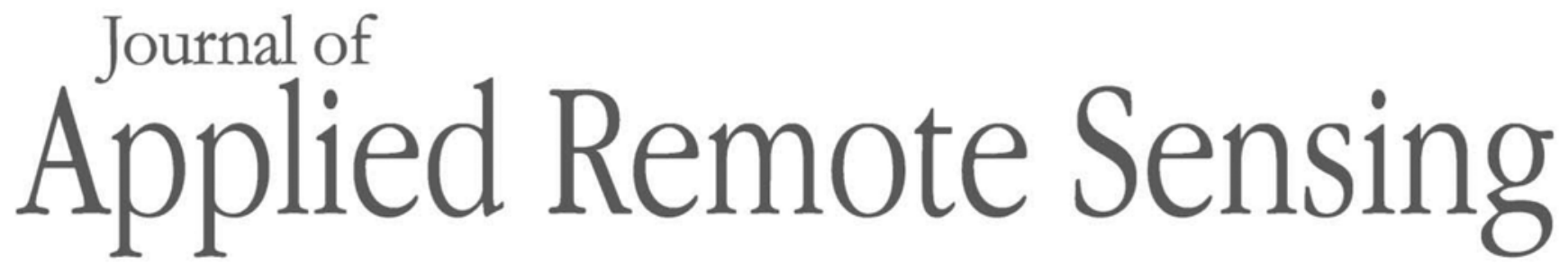

RemoteSensing.SPIEDigitalLibrary.org

\title{
Spectrometers based on acousto-optic tunable filters for in-situ lunar surface measurement
}

\author{
Zhiping He \\ Chunlai Li \\ Rui Xu \\ Gang Lv \\ Liyin Yuan \\ Jianyu Wang
}




\title{
Spectrometers based on acousto-optic tunable filters for in-situ lunar surface measurement
}

\author{
Zhiping He, * Chunlai Li, Rui Xu, Gang Lv, Liyin Yuan, and Jianyu Wang* \\ Chinese Academy of Science, Shanghai Institute of Technical Physics, Key Laboratory of \\ Space Active Opto-Electronics Technology, Shanghai, China
}

\begin{abstract}
The lunar surface consists of rocks of varying sizes and shapes, which are made of minerals, such as pyroxene, plagioclase, olivine, and ilmenite, that exhibit distinctive spectral characteristics in the visible and near-infrared (VIS-NIR) and short-wave infrared (SWIR) regions. To analyze the composition of the lunar surface minerals, several spectrometers based on acousto-optic tunable filters (AOTFs) have been developed to detect lunar surface objects and to obtain their reflectance spectra and geometric images. These spectrometers, including the VISNIR imaging spectrometer onboard China's Chang'e 3/4 unmanned lunar rovers and the Lunar Mineralogical Spectrometer onboard the Chang'e 5/6 lunar landers, use AOTFs as dispersive components. Both are equipped with a VIS/NIR imaging spectrometer, one or several SWIR spectrometers, and a calibration unit with dust-proofing functionality. They are capable of synchronously acquiring the full spectra of the lunar surface objects and performing in-situ calibrations. We introduce these instruments and present a brief description of their working principle, implementation, operation, and major specifications, in addition to the initial scientific achievement of lunar surface exploration. (C) The Authors. Published by SPIE under a Creative Commons Attribution 4.0 Unported License. Distribution or reproduction of this work in whole or in part requires full attribution of the original publication, including its DOI. [DOI: 10.1117/1.JRS.13.027502]
\end{abstract}

Keywords: spectrometer; acousto-optic tunable filter; lunar surface measurement; in situ.

Paper 180880 received Nov. 1, 2018; accepted for publication May 22, 2019; published online Jun. 11, 2019.

\section{Introduction}

Morphological and spectral measurements are the two major methods of analyzing rock structures and compositions. ${ }^{1}$ An imaging spectrometer has the ability to simultaneously obtain both the images and the spectral signatures of targets and is widely used in terrestrial and space-based remote sensing applications. Mineralogical composition analysis is one of the major tasks of China's lunar exploration project. ${ }^{2}$ Minerals such as pyroxene, plagioclase, olivine, and ilmenite, in different sizes and shapes, constitute most of the lunar surface rocks. ${ }^{3}$ The minerals have distinctive spectral characteristics in the VIS/NIR and short-wave infrared (SWIR) wavebands that can be used for identification. Figure 1 shows the spectral reflectance curves of the primary rock-forming single minerals that can be used for mineral identification. ${ }^{4}$

Imaging spectral remote sensing is one of the most important scientific achievements in the past 20 years of the 20th century. Imaging spectrometry combines the imaging technology of traditional remote sensing and spectrum analysis technology. Acousto-optic tunable filter (AOTF), which operates based on acousto-optic interaction, is an electronically tunable optical filter. AOTF has special features compared to other dispersive parts, such as solid state and programmable. An imaging spectrometer based on AOTF is especially suitable for deep space exploration applications (Moon, Mars, and asteroid detection), because of the characteristics such as electronically tunable spectral selectivity, environmental adaptability, rapid response, and simple structure. ${ }^{5-7}$

As early as 1987, a spaceborne spectrometer based on AOTF was developed in the former Soviet Union for ocean exploration. European Space Agency (ESA) successfully launched the Mars Express loaded with the SPICAM (Spectroscopy for Investigation of Characteristics of the Atmosphere of Mars) spectrometer in 2003. ${ }^{8}$ In 2005, ESA Venus Express satellite was also

*Address all correspondence to Zhiping He, E-mail: hzping@mail.sitp.ac.cn; Jianyu Wang, E-mail: jywang@mail.sitp.ac.cn 


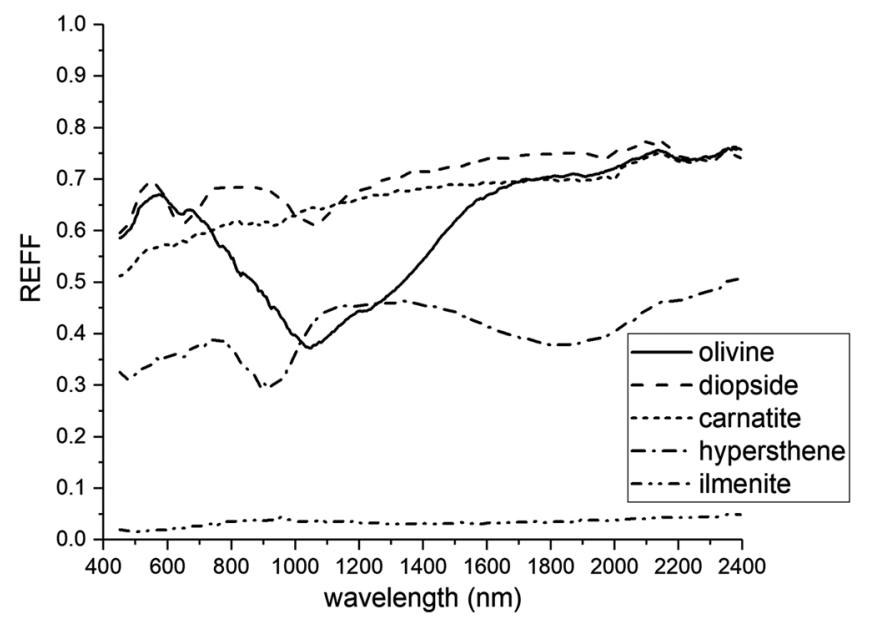

Fig. 1 Spectral reflectance curves for the main single minerals on the lunar surface.

loaded with AOTF spectrometer for Venus detection. ${ }^{9}$ Since 2006, the Shanghai Institute of Technical Physics began to study imaging spectrometers based on AOTF. Visible and nearinfrared imaging spectrometer (VNIS) is a payload of lunar rover for 0.45 - to $2.4-\mu \mathrm{m}$ spectral bands detection and will be able to inspect and probe minerals in the rover region. Lunar mineralogical spectrometer (LMS) is a payload integrated with a scanning module and spectral programmable imaging spectrometer for a future lunar lander.

\section{Spectrometers Based on AOTFs}

\subsection{Visible and Near-Infrared Imaging Spectrometer}

The VNIS is one of the main scientific payloads on the lunar rovers for Chang'e 3 and Chang'e 4. It uses AOTFs as the dispersive components. ${ }^{10,11}$ The VNIS mostly addresses the lunar surface material composition and available resource exploration for determining the lunar surface mineral composition and performing a comprehensive analysis of the chemical compositions. The VNIS is mounted on the platform of the lunar rover and detects the spectra and images of the lunar objects in the roving area to provide scientific data. ${ }^{9}$ As a passive optical instrument, the VNIS measures the radiance diffusely reflected from solar illumination of the Moon's surface. It includes two detection channels: a VIS/NIR channel $(0.45$ to $0.95 \mu \mathrm{m})$ with a CMOS area array detector and a SWIR channel ( 0.9 to $2.4 \mu \mathrm{m}$ ) with an InGaAs single-element detector. Each channel consists of image-forming lens, field stop, collimating lens, convergent lens, and the AOTF. Furthermore, a calibration unit with dust-proofing functionality, motor drive circuit, radio frequency (RF) drive circuits, and main control circuits has been integrated in this payload as the public component, as shown in Fig. 2. The structure, optical module, and design of the VNIS are detailed in Figs. 3 and 4.

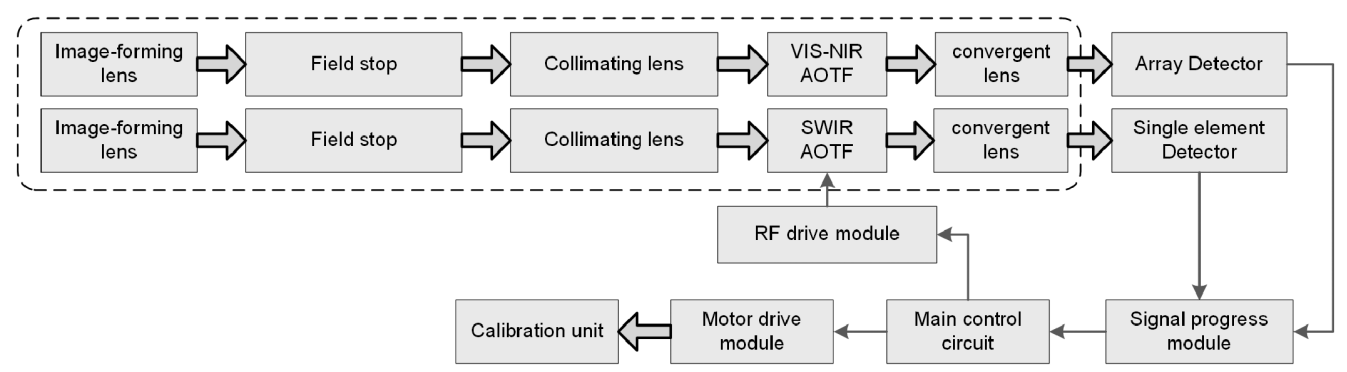

Fig. 2 Schematic diagram of the optical, electronic, and calibration modules of the VNIS. 

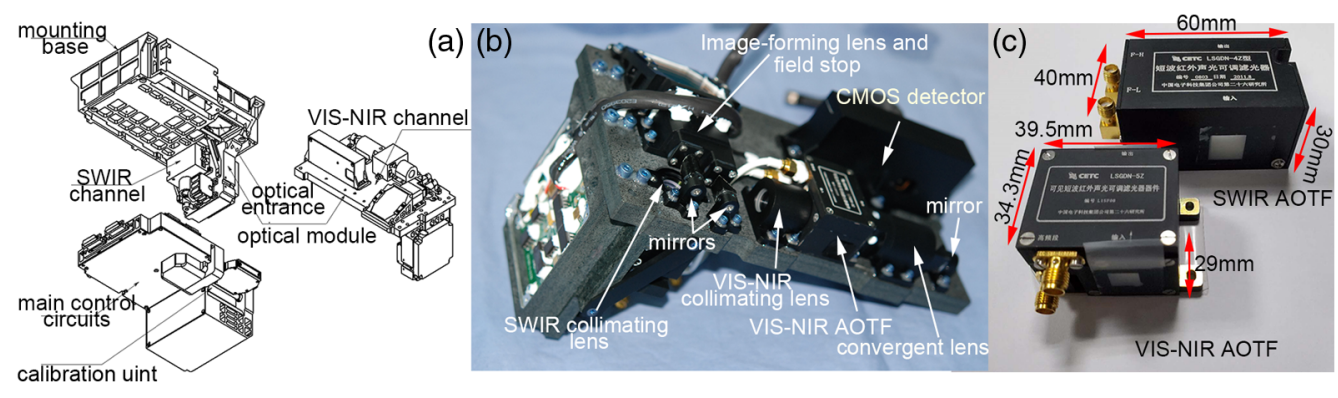

Fig. 3 (a) Assembly diagram of the VNIS, (b) the optical modules, (c) AOTFs used in VNIS; the aperture of visible and near-infrared (VIS-NIR) AOTF is $8 \times 8 \mathrm{~mm}^{2}$, and the SWIR one is $12 \times 12 \mathrm{~mm}^{2}$

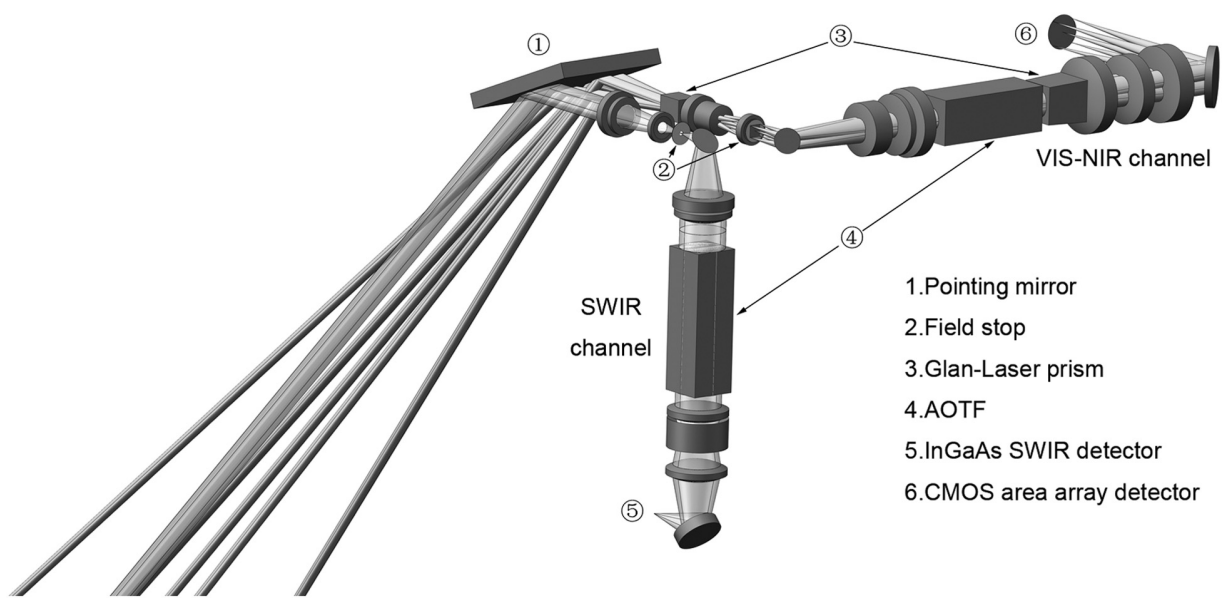

Fig. 4 Diagram of the optical design of the VNIS.

As shown in Fig. 4, two types of AOTFs have been used in the optical system. Each AOTF has two transducers with separate ports to optimize the bandpass (FWHM) and diffraction efficiency. Furthermore, an optical wedge has been designed at the exit port to eliminate chromatic aberration in both AOTFs. The main characteristics of the VNIS AOTFs are shown in Table 1. The performance of AOTFs such as acoustic frequency range and diffraction efficiency is shown in Fig. 5.

After its soft landing on the Moon, Chang'e 3 carried out a lunar survey followed by scientific exploration activities. The VNIS mostly analyzed the lunar surface material composition

Table 1 Main characteristics of the VNIS AOTFs.

\begin{tabular}{lcc}
\hline \hline & VIS-NIR AOTF & SWIR AOTF \\
\hline Material & $\mathrm{TeO}_{2}$ & \\
Spectral range & 0.45 to $0.95 \mu \mathrm{m}$ & 0.9 to $2.4 \mu \mathrm{m}$ \\
FWHM & 2.0 to $6.0 \mathrm{~nm} @<633 \mathrm{~nm}$ & 3.0 to $6.0 \mathrm{~nm} @<1380 \mathrm{~nm}$ \\
& 2.5 to $7.0 \mathrm{~nm} @>633 \mathrm{~nm}$ & 4.0 to $12.0 \mathrm{~nm} @>1380 \mathrm{~nm}$ \\
Acoustic frequency range & 70.7 to $178.6 \mathrm{MHz}$ & 41.9 to $118.9 \mathrm{MHz}$ \\
Angular aperture & $>7 \mathrm{deg}$ & $>8 \mathrm{deg}$ \\
Diffraction angle & $>5.6 \mathrm{deg}$ & $>7.5 \mathrm{deg}$ \\
Power & & $\sim 2 \mathrm{~W}$ \\
\hline \hline
\end{tabular}



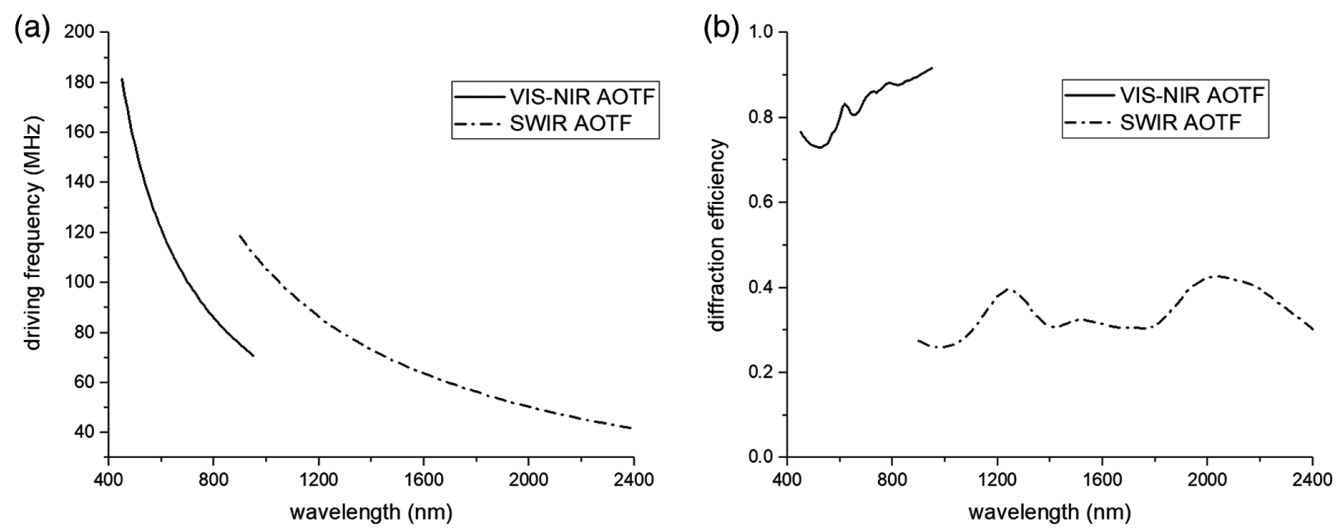

Fig. 5 The performance of AOTFs equipped in VNIS. (a) The relationship between wavelength and driving frequency and (b) the diffraction efficiency of the VIS-NIR AOTF and SWIR AOTF.
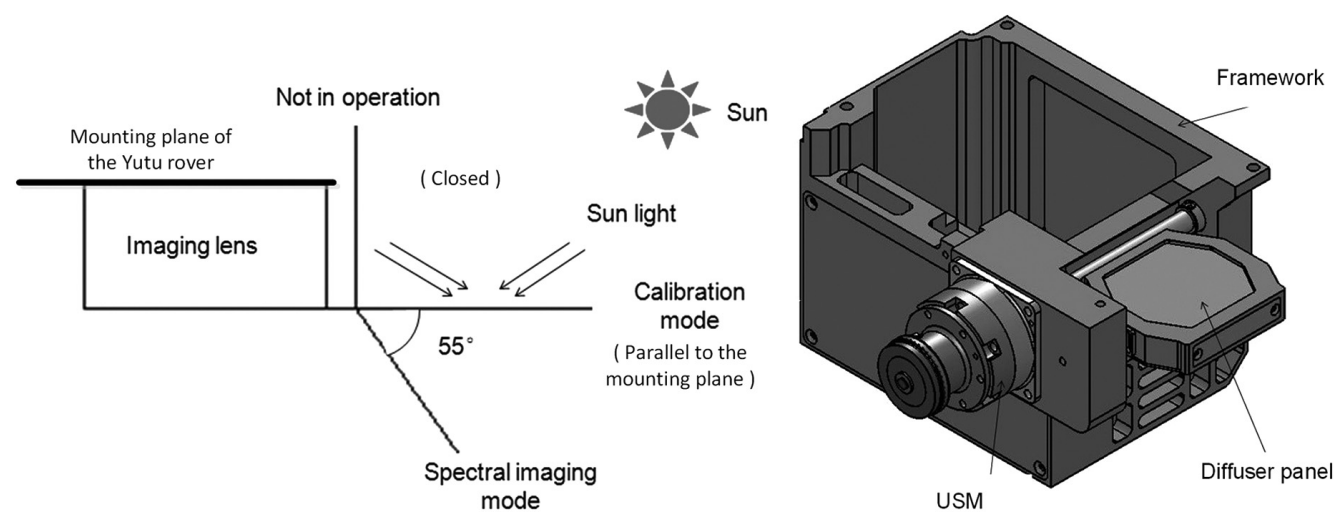

Fig. 6 Schematic view of the structure and functioning of the calibration unit.

and explored the available resources. The VNIS operated in two modes: detection and calibration. In the detection mode, the VNIS acquired scientific data from the lunar surface objects; the angle between the framework of the calibration unit and the horizontal mounting face of VNIS was about $55 \mathrm{deg}$. In the calibration mode, using solar radiation as the calibration source, the diffusing calibration plate of the calibration unit was set to a position parallel to the mounting plane to allow calibration of the instrument. The calibration unit of the VNIS at the light entrance consisted of an ultrasonic motor (USM), harmonic reducer, framework, and an internal diffuser panel. The operating modes and dust-proofing function were realized by the self-locking characteristics of the USM. ${ }^{10}$ The structure and function of the calibration unit are shown in Fig. 6.

The VNIS was mounted in front of the lunar rover to detect lunar surface objects at a 45-deg viewing angle and to obtain the spectra and geometric data at a height of $0.695 \mathrm{~m}$, as shown in Fig. 7.

\subsection{Lunar Mineralogical Spectrometer}

The LMS is one of the main scientific payloads onboard the lunar landers for Chang'e 5 and Chang'e 6. The LMS primarily follows the technology of the VNIS but expands its spectral range from 0.48 to $3.2 \mu \mathrm{m}$. It has the following functions: (1) to obtain the spectral image data of the specified objects in the VIS-NIR and spectral data in the SWIR channels, (2) to perform in-situ calibration, and (3) spectral imaging and spectral detection with multiband in full scanning region.

The LMS includes four detection channels: one is a hyperspectral imager with a spectral range of 0.48 to $0.95 \mu \mathrm{m}$, and the others are three spectrographs and their total spectral range being 0.9 to $3.2 \mu \mathrm{m}$. The LMS includes a two-dimensional (2-D) scanning mechanism, imageforming lens, collimating lens, AOTF, convergent lens, the detector components, motor drive, 

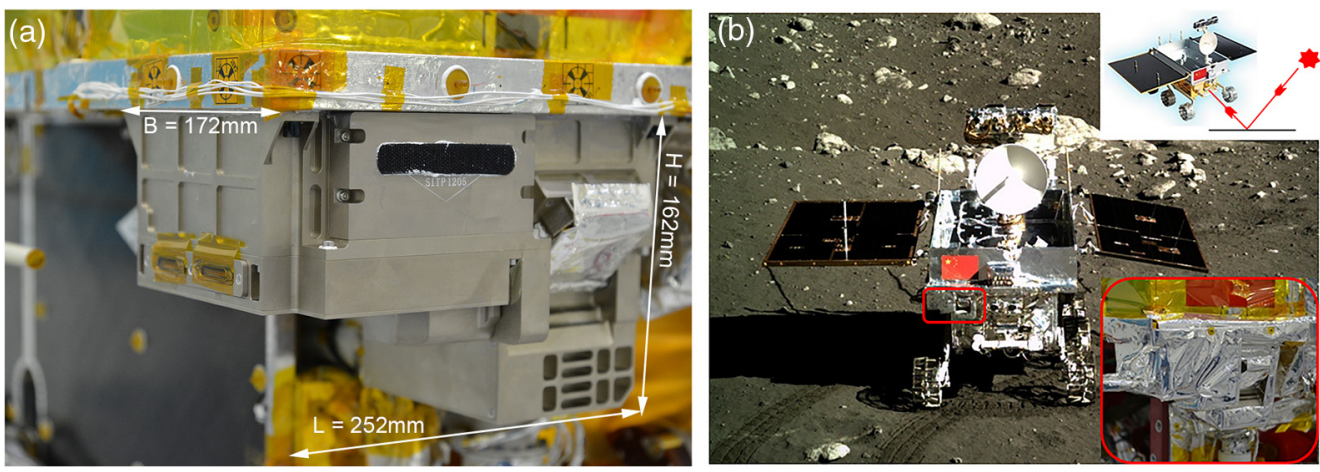

Fig. 7 (a) Structural dimensions and mounting position of the VNIS. (b) The photograph of the Yutu lunar rover taken by the terrain camera (TCAM) at point A of the soft landing site.

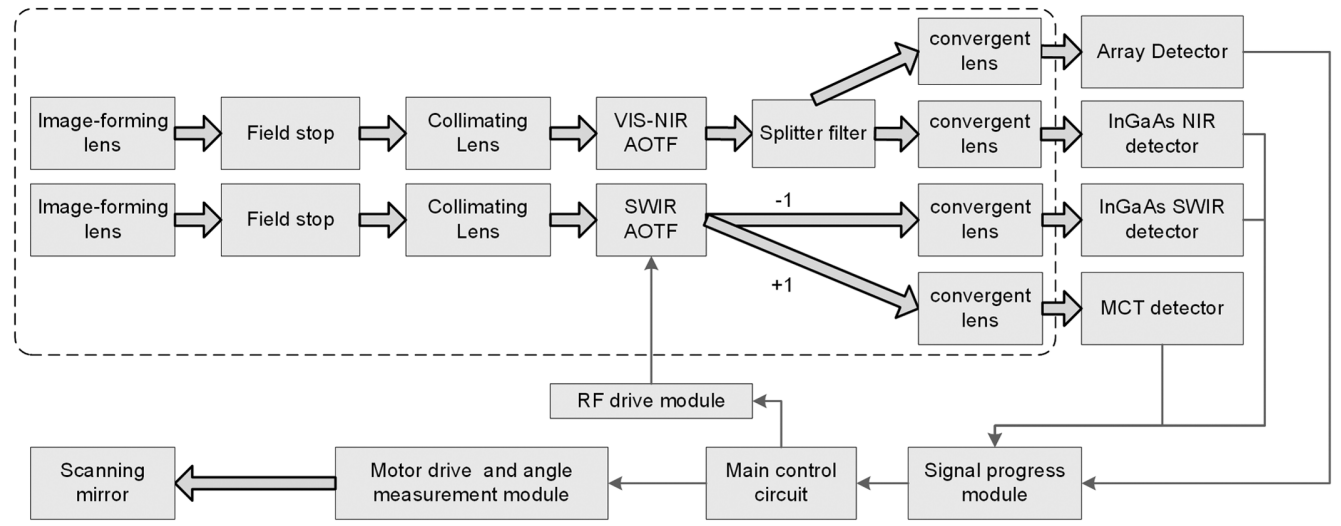

Fig. 8 System diagram of LMS.

RF drive, and a main control circuit, as shown in Fig. 8. It is a kind of scanning, spectral programmable imaging spectrometer and includes a scanning mechanism and a programmable spectral selection spectrometer.

As shown in Fig. 9, two AOTFs have been used in the optical system. They also have ports to optimize the bandpass (FWHM) and diffraction efficiency. The -1 order diffraction light of the VIS-NIR AOTF was used for the VIS spectral imaging and NIR spectra acquirement, where an

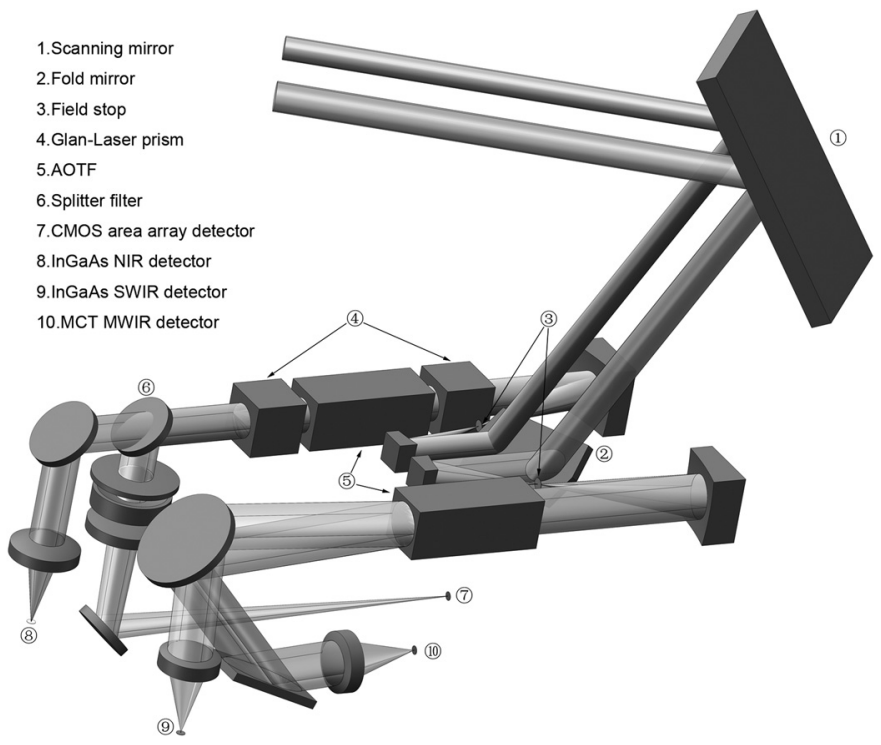

Fig. 9 Diagram of the optical design of LMS. 
He et al.: Spectrometers based on acousto-optic tunable filters for in-situ...

Table 2 Main characteristics of the VNIS AOTFs.

\begin{tabular}{lcc}
\hline \hline & VIS-NIR AOTF & IR AOTF \\
\hline Material & $\mathrm{TeO}_{2}$ & \\
Spectral range $(\mu \mathrm{m})$ & 0.48 to 1.45 & 1.4 to 3.2 \\
FWHM & 2.6 to $9.4 \mathrm{~nm} @<780 \mathrm{~nm}$ & 7.6 to $20.8 \mathrm{~nm} @ 1.4-2.3 \mu \mathrm{m}$ \\
& 2.4 to $9.0 \mathrm{~nm} @>780 \mathrm{~nm}$ & 11.6 to $24.9 \mathrm{~nm} @ 2.2$ to $3.2 \mu \mathrm{m}$ \\
Acoustic frequency range $(\mathrm{MHz})$ & 45.2 to 163.6 & 27.7 to 66.2 \\
Angular aperture $(\mathrm{deg})$ & $>7$ & $>3$ \\
Diffraction angle $(\mathrm{deg})$ & $>5.9$ & $>6.5$ \\
Power $(\mathrm{W})$ & & \\
\hline \hline
\end{tabular}

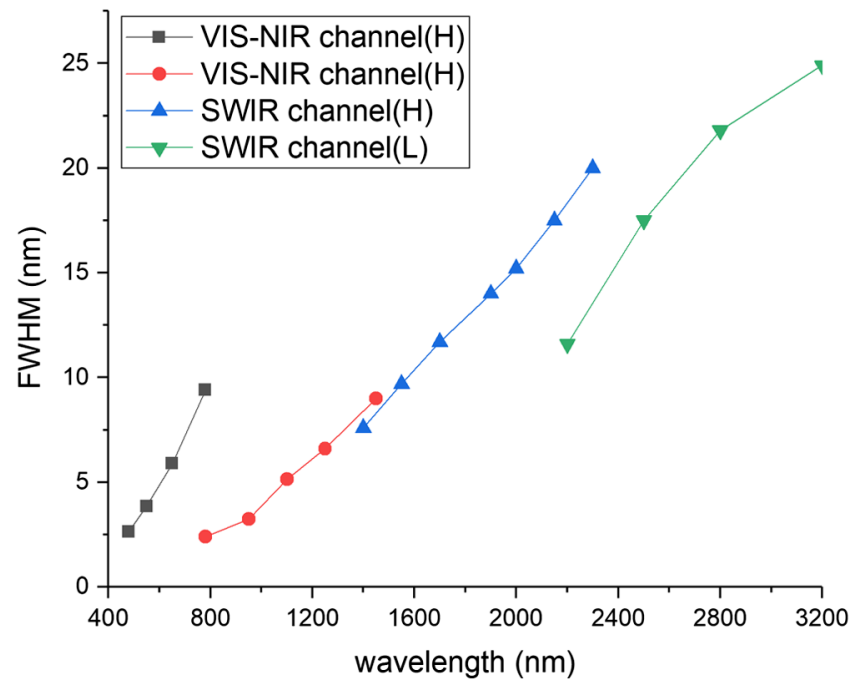

Fig. 10 Spectral resolution of LMS, separated into four channels by AOTF ports.

optical wedge was designed for the AOTF to eliminate chromatic aberration. The \pm 1 order diffraction lights of the IR AOTF were used for the SWIR and MWIR channels, respectively. The main characteristics of these AOTFs are shown in Table 2.

After spectral calibration, the spectral resolution was 2.4 to $9.4 \mathrm{~nm}$ in the VIS-NIR and 7.6 to $24.9 \mathrm{~nm}$ in the IR channels, as shown in Fig. 10.

Images of the lander and LMS are shown in Fig. 11. The LMS is mounted on the $+Z-Y$ panel of the lander to detect the lunar surface objects and obtain the spectra and geometric data at a height of about $1.55 \mathrm{~m}$. Because the platform on which the LMS is mounted is unmovable, a pointing component is required for expanding the observable areas. The 2-D scanning mechanism includes USMs, harmonic reducer, rotary potentiometers, and an aluminum-based scanning mirror, which ensure that the system is light and minimized and the system can adapt to a high surrounding temperature of up to $94^{\circ} \mathrm{C}$.

\section{Main Characteristics and Primary Detection Results}

\subsection{Visible and Near-Infrared Imaging Spectrometer}

The VNIS can simultaneously obtain a spectral image in the VIS/NIR channel and spectral data in the SWIR channel. The optical axis of the VIS/NIR and SWIR channels are parallel to each 


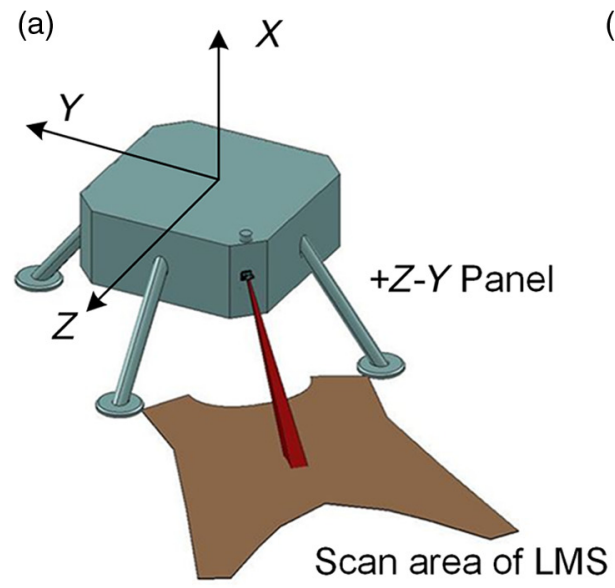

(b)

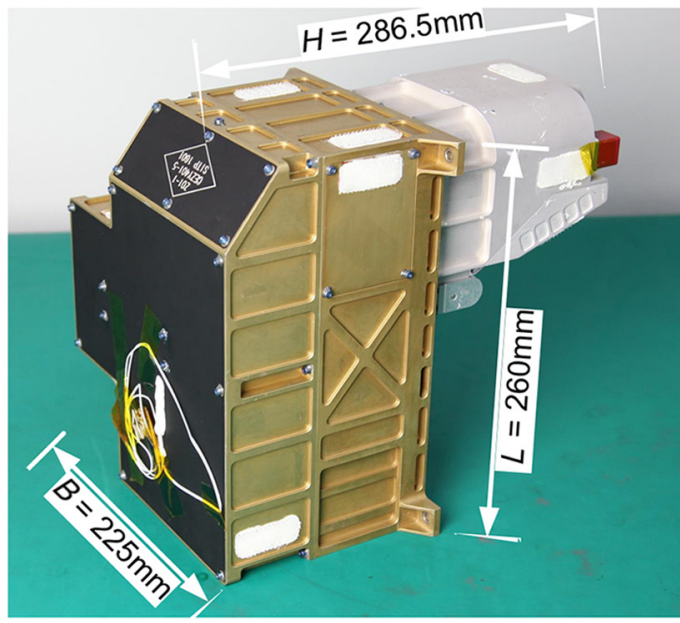

Fig. 11 Schematic diagram of Chang'e 5 mission: (a) mounting position of the LMS on the lander of Chang'e 5 probe and (b) the flight product of the LMS.

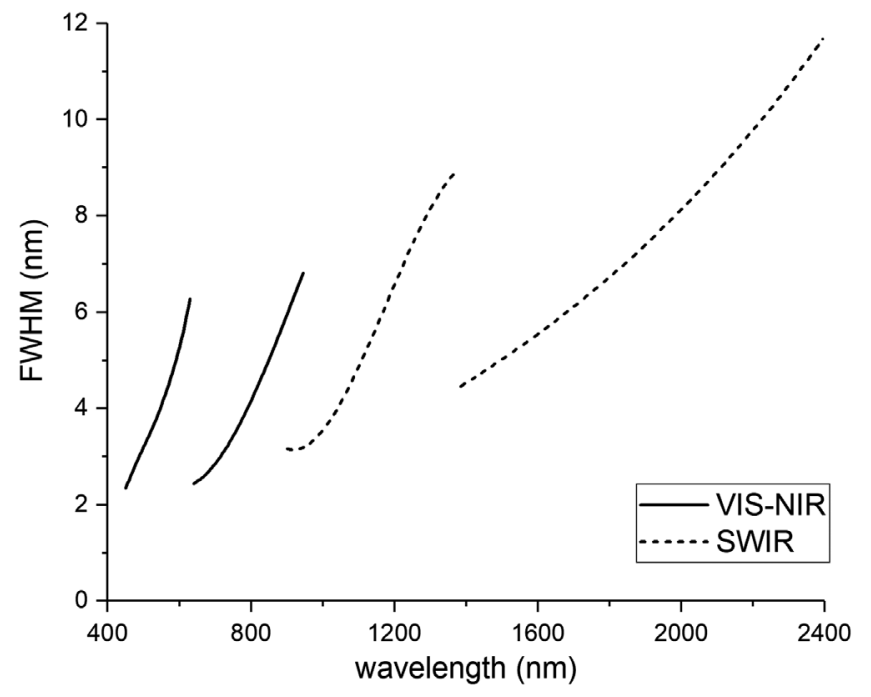

Fig. 12 Spectral resolution of VNIS/Yutu, separated into four channels by AOTF ports.

other at a distance of $18 \mathrm{~mm}$. The fields of view (FOVs) in the VIS/NIR and SWIR channels are $8.5 \mathrm{deg} \times 8.5 \mathrm{deg}$ and $\Phi 3.6 \mathrm{deg}$, respectively. According to the spectral calibration, the spectral ranges of the VNIS are 449 to $950 \mathrm{~nm}$ with 2- to 7-nm resolution in the VIS/NIR channel and 899 to $2402 \mathrm{~nm}$ with 3- to 12-resolution in the SWIR channel, which are shown in Fig. 12.

The signal-to-noise ratio (SNR) of the VNIS is tested and analyzed by ground radiometric calibration. The SNR changes with the input signal, and it is an important indicator of the radiometric response characteristics of the VNIS. The SNR is $>31 \mathrm{~dB}$ in the VIS-NIR band when the albedo is 0.09 and the solar incident angle is $45 \mathrm{deg}$; and the SNR is $>32 \mathrm{~dB}$ in the SWIR channel when the albedo is 0.09 and the solar incident angle is $75 \mathrm{deg} .{ }^{10}$ The main performance specifications of the VNIS are shown in Table 3.

After the Chang'e 3 mission began its scientific exploration stage, the VNIS instrument successfully completed the first lunar surface spectral acquisition at BTC 10:10 on December 23, 2013. During the first two lunar days, the VNIS performed measurements at four locations and obtained data in detection mode (four times) and calibration mode (three times). The total size of data was $350 \mathrm{MB} .{ }^{11}$ The topographical images of N205 and the REFF of all detection nodes are shown in Fig. 13.

During the Chang'e-4 mission, VNIS was carried on Yutu-2 lunar rover. Since the successful soft landing in Von Kármán crater on the far side of the Moon on January $32019,{ }^{12}$ VNIS 
He et al.: Spectrometers based on acousto-optic tunable filters for in-situ...

Table 3 Main performance specifications of VNIS/Yutu. ${ }^{10}$

\begin{tabular}{|c|c|c|}
\hline \multirow[b]{2}{*}{ Description } & \multicolumn{2}{|c|}{ Chang'e 3's specification performances } \\
\hline & VIS-NIR & SWIR \\
\hline Spectral coverage $(\mathrm{nm})$ & 449 to 950 & 899 to 2402 \\
\hline Spectral resolution $(\mathrm{nm})$ & 2 to 7 & 3 to 12 \\
\hline FOV (deg) & $8.5 \times 8.5$ & $\Phi 3.6 \mathrm{deg}$ \\
\hline Effective pixels & $256 \times 256$ & 1 \\
\hline Quantization (bits) & 10 & 16 \\
\hline SNR (dB) & $\begin{array}{l}\geq 31 \text { (albedo is } 0.09 \text { and solar } \\
\text { incident angle is } 45 \text { deg) }\end{array}$ & $\begin{array}{l}\geq 32 \text { (albedo is } 0.09 \text { and solar } \\
\text { incident angle is } 75 \mathrm{deg} \text { ) }\end{array}$ \\
\hline Spectral sampling interval (nm) & 5 & \\
\hline Power consumption (W) & 19.8 & \\
\hline Weight (kg) & $\begin{array}{l}4.7 \text { (spectrometer probe) } \\
0.7 \text { (logical control component in } \\
\text { Rover payload Electric Control Box) }\end{array}$ & \\
\hline
\end{tabular}

(a)

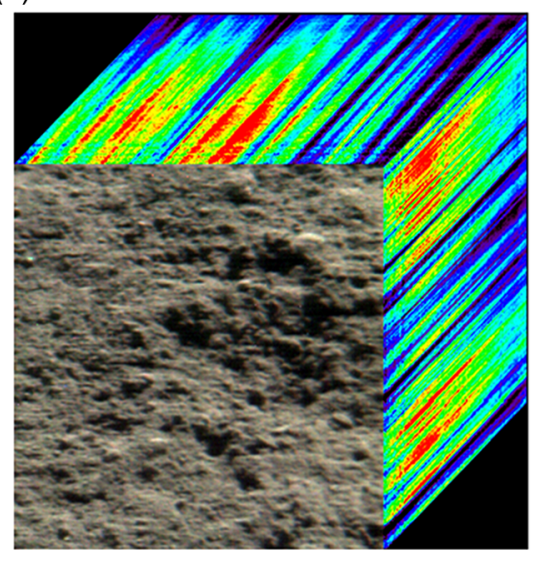

(b)

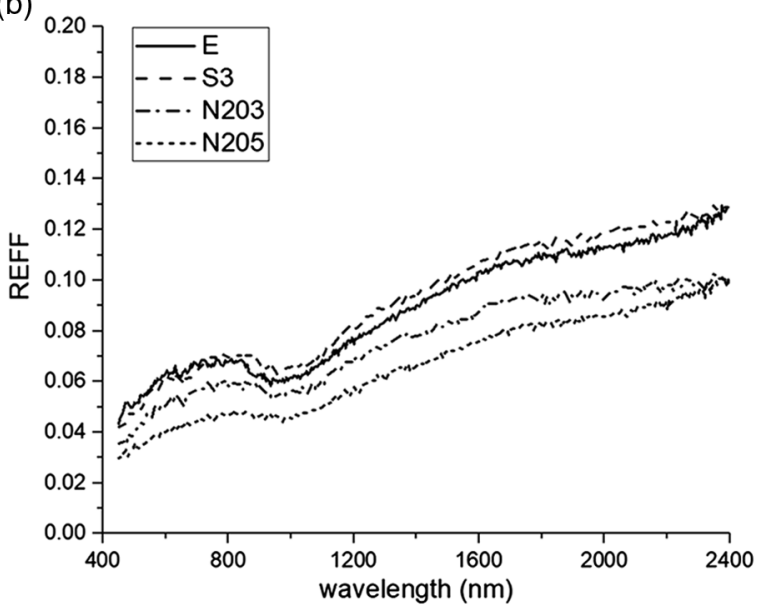

Fig. 13 (a) Data from VNIS/Yutu at N205 note, false color picture (500, 550, and $645 \mathrm{~nm}$, in the right) and (b) REFF curves of four lunar exploration nodes during two lunar days by VNIS/Yutu.

had been working for five lunar days and performed 27 detections. The selected targets such as Yutu-2's wheel track and rock, obtained in the detection node A and LE00303, and their corresponding REFF features are shown in the Fig. 14.

\subsection{Lunar Mineralogical Spectrometer}

In the detection mode, the LMS obtained spectral images in the VIS/NIR channel and spectral data in three SWIR channels in sequence. The main performance specifications of the LMS are shown in Table 4.

Performance parameters such as FOV, spectral range, spectral resolution, radiance, and geometric imaging features were calibrated in the ground experiment. The FOV of the LMS was $4.17 \mathrm{deg} \times 4.17 \mathrm{deg}$ in all channels.

The SNR is $>34 \mathrm{~dB}$ in the VIS/NIR band, and $>39 \mathrm{~dB}$ in the IR band, as shown in Fig. 15 . 
(a)

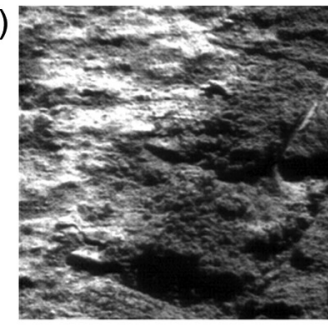

(b)

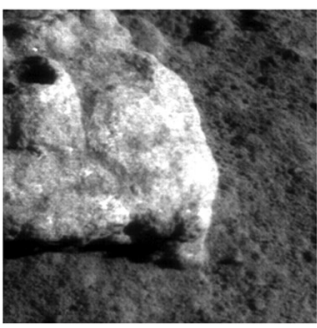

(c)

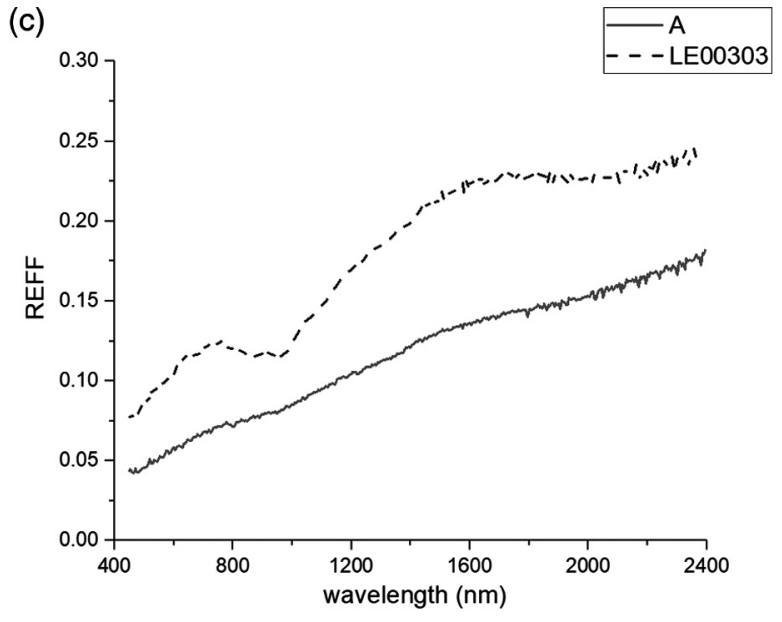

Fig. 14 (a) A 635-nm image from VNIS/Yutu-2 at node A, (b) 635-nm image from VNIS/Yutu-2 at node LE00303, and (c) REFF curves of nodes A and LE00303.

Table 4 Main performance specifications of the LMS.

\begin{tabular}{|c|c|c|c|c|c|}
\hline \multirow[b]{2}{*}{ Description } & \multicolumn{2}{|c|}{ Specification requirements } & \multicolumn{3}{|c|}{$\begin{array}{l}\text { Chang'e 5's specification } \\
\text { performances }\end{array}$} \\
\hline & VIS-NIR & SWIR & & VIS-NIR & SWIR \\
\hline Spectral coverage $(\mathrm{nm})$ & 480 to 950 & 900 to 3200 & & 479 to 955 & 896 to 3212 \\
\hline Spectral resolution $(\mathrm{nm})$ & 5.0 to 25.0 & 5.0 to 25.0 & & 2.4 to 9.4 & 7.6 to 24.9 \\
\hline FOV (deg) & $\geq 3.0 \times 3.0$ & $\geq 3.0 \times 3.0$ & & $4.17 \times 4.17$ & $4.17 \times 4.17$ \\
\hline Effective pixels & $256 \times 256$ & 1 & & $256 \times 256$ & 1 \\
\hline Quantization (bits) & $\geq 10$ & $\geq 10$ & & 10 & 16 \\
\hline $\begin{array}{l}\text { SNR (dB, albedo is } 9 \% \text { and solar } \\
\text { incident angle is } 45 \mathrm{deg} \text { ) }\end{array}$ & $\geq 30$ & $\geq 30$ & & $\geq 34$ & $\geq 39$ \\
\hline Spectral sampling interval (nm) & \multicolumn{2}{|c|}{5} & & \multicolumn{2}{|c|}{5} \\
\hline Power consumption (W) & \multicolumn{5}{|c|}{16.0} \\
\hline Weight $(\mathrm{kg})$ & \multicolumn{5}{|c|}{5.6} \\
\hline
\end{tabular}

\section{Conclusions}

To analyze the composition of the lunar surface minerals, several spectrometers based on AOTFs have been developed to detect lunar surface objects and to obtain their reflectance spectra and geometric images; these include the VNIS onboard China's Chang'e 3 and Chang'e 4 lunar rovers and the LMS onboard Chang'e 5 and Chang'e 6 lunar landers. These spectrometers are capable of synchronously acquiring the full spectra of the lunar surface objects and performing in-situ calibrations. The VNIS/Yutu has performed several explorations and calibrations, and obtained several spectral images and spectral reflectance curves of the lunar soil in the Imbrium region following its first successful operation at the landing site on December 23, 2013. The VNIS/Yutu-2, which is travelling in Von Kármán crater after the soft landing on January 3, 2019, ${ }^{12}$ will provide valuable in-situ hyperspectral images and infrared spectra of the soil, rocks, and other materials on the far side of the Moon. The flight module of LMS has completed all the ground calibration and environmental verification tests and will 


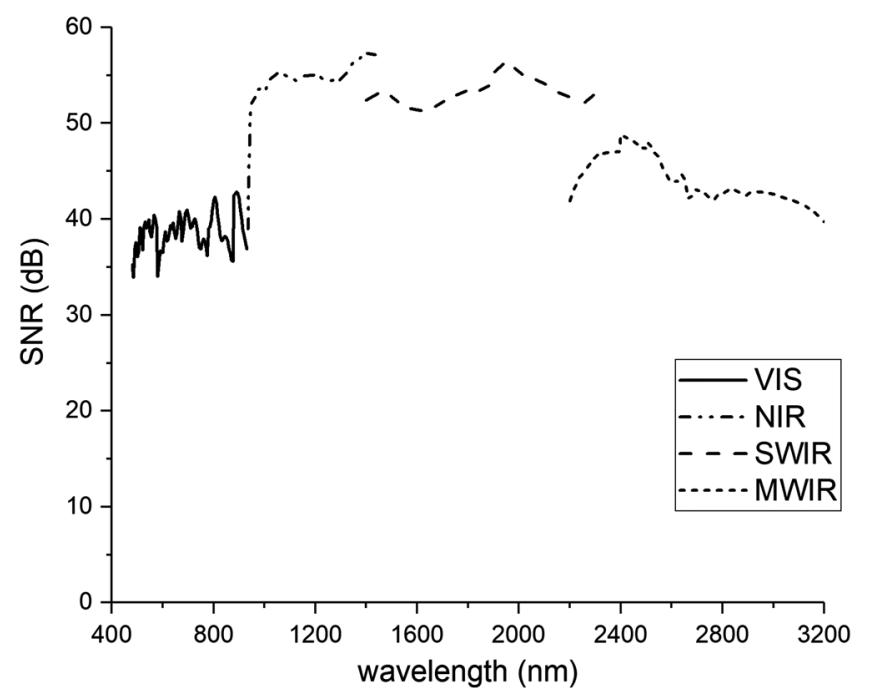

Fig. 15 SNR of LMS. The performance of LMS is calculated by the radiance calibration at the typical temperature $\left(20^{\circ} \mathrm{C}\right)$.

observe the sampling area in the lunar surface sampling mission with the Chang'e 5 probe by the end of 2019.

\section{Acknowledgments}

This paper was supported by the special funds for the second and third phases of the Chinese lunar exploration program, the National Natural Science Foundation (Nos. 21105109 and 61605231), and the Program of Shanghai Academic/Technology Research Leader (No. 19XD1424100). The authors thank the Science and Application Center for the Moon and Deep Space Exploration of the Chinese Academy of Sciences for the ground test and data preprocessing and the National Space Science Center of the Chinese Academy of Sciences for its contributions to the development and testing of the instruments.

\section{References}

1. Z.-P. He et al., "Operating principles and detection characteristics of visible and nearinfrared imaging spectrometer (VNIS) in Chang'e 3," Res. Astron. Astrophys. 14(12), 1567-1577 (2014).

2. P. J. Ye and J. Peng, "Deep space exploration and its prospects in China," Eng. Sci. 8(10), 13-18 (2006).

3. B. Liu et al., "Reflectance conversion methods for the VIS/NIR imaging spectrometer aboard the Chang'E-3 lunar rover: based on ground validation experiment data," Res. Astron. Astrophys. 13(7), 862-874 (2013).

4. Z. He et al., "Visible and near-infrared imaging spectrometer (VNIS) and its preliminary results from the Chang'E 3 Project," Rev. Sci. Instrum. 85, 083104 (2014).

5. O. Korablev et al., "Development of a mast or robotic arm-mounted infrared AOTF spectrometer for surface Moon and Mars probes," Proc. SPIE 9608, 960807 (2015).

6. J.-P. Bibring et al., "The MicrOmega investigation onboard Hayabusa2," Space Sci. Rev. 208, 401-412 (2017).

7. Zh. He et al., "Visible and near-infrared imaging spectrometer (VNIS) for Chang'E-3," Proc. SPIE 9263, 92630D (2014).

8. J. Wang et al., "Visible and near-infrared imaging spectrometer aboard Chinese Chang'E-3 spacecraft," in Optical Payloads for Space Missions, Ch. 5, S-E Qian, Ed., John Wiley \& Sons, Chichester (2015). 
9. J.-L. Bertaux et al., "SPICAV on Venus Express: three spectrometers to study the global structure and composition of the Venus atmosphere," Planet. Space Sci. 55(12), 1673-1700 (2007).

10. Zh. He et al., "Visible and near-infrared imaging spectrometer (VNIS) for in-situ lunar surface measurement," Proc. SPIE 9639, 96391S (2015).

11. Zh. He et al., "The spectrometers based on AOTF for in-situ Lunar surface measurement," Proc. SPIE 10780, 107800N (2018).

12. C. Li et al., "Chang'E-4 initial spectroscopic identification of lunar far-side mantle-derived materials," Nature 569, 378-382 (2019).

Zhiping He is a professor in Shanghai Institute of Technical Physics of the Chinese Academy of Sciences. He has long been engaged in the research of space optics and optoelectronic technology and has participated and completed the development of Chang'e 3 visible and near-infrared imaging spectrometer (VNIS). Currently, he is conducting several researches on the spectral detection payloads, which are used for the exploration of Moon and Mars. The representative work includes Chang'e 5 lunar mineralogy spectrometer (LMS) and Mars mineralogy spectrometer (MMS).

Biographies of the other authors are not available. 